\title{
Kompetenzen statt Weiterbildungszeiten?
}

Szene 1: Frisch inspiriert von der letzten Facharztprüfung, die ich abnehmen durfte, frage ich mich, ob wir diese Prüfungen richtig gestalten. Ich kann mich an meine eigene Prüfung erinnern, schon ein paar Tage her - zugegeben. Der anwesende Psychiater hat mich in der neurologischen Facharztprüfung gut 20 Minuten über Pharmakodynamik und Pharmakokinetik von Neuroleptika und Antidepressiva befragt. Freundlich zugewandt, aber doch sehr gründlich - im Studium hätte ich das besser gewusst als am Ende der Weiterbildung zum Facharzt, aber es hat dann wohl gerade gereicht. Mit dem prüfenden Neurologen war es ein angemessenes, durchaus detailliertes medizinisches Fachgespräch, das im „Anton-Syndrom“ endete, eingeleitet durch eine kurze Bemerkung des Prüfers, ob ich denn Skifahren würde und falls ja, würde mir das Syndrom auch gleich einfallen. Auch Skifahren ist eine Kompetenz. Hätte ich die Prüfung direkt nach dem Studium auch bestanden? Würde ich nicht ausschließen. Aber da sind ja noch die Zahlen und Weiterbildungszeiten, die das unmöglich machen. Sie gelten auch als Surrogatparameter für die erworbenen Kompetenzen, die einen Neurologen ausmachen sollten. Genau um das Thema „Kompetenzen“ ging es auch beim diesjährigen Deutschen Ärztetag (Drucksache III - 17; 119. Deutscher Ärztetag 2016). Der Deutsche Ärztetag fordert die Bundesärztekammer auf, „,bei der Novellierung der (Muster-)Weiterbildungsordnung (MWBO) die geplanten vorgegebenen starren Weiterbildungszeiten zugunsten der Feststellung von Kompetenzen zu überprüfen“. Also Direktmessung statt Surrogatparameter? Und wie?

Szene 2: Unlängst hielten wir eine $\mathrm{PhD}$-Prüfung bei einer NichtMedizinerin ab. Sehr gute Dissertation, drei Publikationen, exzellenter Vortrag, souveräne Diskussion. In der Prüfungskommission war ein externer Professor aus den Benelux-Staaten. Eigentlich eine klare Sache. Nach Jahren harter Arbeit der Kandidatin und einer sehr guten schriftlichen Leistung "gefühlt“ Zeit für eine wissenschaftliche Diskussion auf Augenhöhe. Aber der Kollege aus dem Ausland brachte die Kandidatin ordentlich an ihre Grenzen, nicht so sehr im Sinne von „Augenhöhe“, „kollegialem Fachgespräch “ und einer Abrundung der bisherigen tollen Leistungen, sondern im Sinne von Prüfung und schonungsloser wissenschaftlicher Analyse: „Haben Sie darüber nachgedacht? Wieso haben Sie nicht ...? Was werden Sie mit den Erfahrungen der Dissertation bei den Folgeexperimenten anders machen?" - und so weiter. Danach war alles klar, alle Prüfer überzeugt, die Note super, alles gut. Nota bene: Zwar blitzte mehrfach das Erkennen der eigenen Grenzen in den Augen der Probandin auf, dennoch war sie es letztlich, die sich am Abschluss ihrer Dissertation nochmals beweisen konnte. Ein Erfolgserlebnis und ein rigoroses Rigorosum.

Epilog: Ein kollegiales Gespräch auf Augenhöhe ist als Prüfung nach sechsjähriger Weiterbildung stilistisch angemessen, aber wir müssen den Probanden auch die Chance geben, überdurchschnittliche Leistungen zu beweisen. Dafür ist an die Grenzen zu gehen - freundlich, aber bestimmt. Wenn zur Erlangung eines akademischen Grades wie Dr. med. oder $\mathrm{PhD}$ eine rigorose Prüfung angemessen ist, dann muss das auch für die Lizenz zur eigenverantwortlichen fachärztlichen Versorgung von Patienten gelten. Es ist auch keine Schande, wenn man aus einer

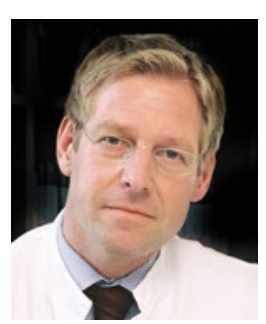

Prof. Dr. med. Christian Gerloff

Klinik und Poliklinik für Neurologie

Kopf- und Neurozentrum

Universitätsklinikum Hamburg-Eppendorf

Martinistr. 52, 20246 Hamburg

E-Mail: gerloff@uke.de

bestandenen Prüfung mit einigen klaren Vorschlägen herausgeht, an was man noch arbeiten sollte.

Können wir auf Zahlen und Weiterbildungszeiten verzichten? Nein. Sie sind überprüfbar und geben nicht zuletzt auch den Weiterbildungsassistenten ein Instrument an die Hand, um Ausbildungsinhalte einzufordern. Wie gut sind sie als Surrogatparameter für Kompetenzen? Der Erwerb klinisch-neurologischer Kompetenzen muss mehr in den Vordergrund der Überprüfung rücken. Mittlerweile ersetzt ein Tabellenwerk das frühere individualisierte Facharztzeugnis, in dem viel mehr Raum war, sich zu Kompetenzen der Kandidaten zu äußern. Ich plädiere schon jetzt dafür, dass zusätzlich zum Tabellenwerk die Weiterbildungsbefugten auch künftig solch individuelle Zeugnisse abfassen, sich dort dezidiert zu den Kompetenzen der Kandidaten äußern und dass diese Schriftstücke mit den Prüfungsunterlagen bei der zuständigen Ärztekammer eingereicht werden.

Sollte man Kompetenzen praxisnäher prüfen? Es muss erlaubt sein, über einen praktischen Teil der Facharztprüfung nachzudenken. Ich weiß, das gab es schon - aber wenn wir es logistisch schaffen, die Studierenden praktisch und theoretisch zu prüfen, warum nicht auch die Facharztanwärter? Auch Kompromisse sind denkbar, wie die Vorlage eines detailliert ausgearbeiteten Falles, den die Kandidaten vorstellen und den man zusätzlich zum heute üblichen Prüfungsablauf kritisch diskutiert. Ich finde es sehr zu begrüßen, dass Deutscher Ärztetag und Bundesärztekammer dieses Thema (für alle Disziplinen) angehen wollen.

Die Neurologie entwickelt sich dynamisch, sowohl in der Tiefe als auch in der Breite. Vielerorts klagen Weiterbildungsassistenten darüber, dass sie vor allem in der neurologischen Akutmedizin eingesetzt sind: Notaufnahme, Stroke Unit, Intensivstation, Schichtbetrieb. Häufig zu kurz kommen Differenzialdiagnostik komplexer, einschließlich neurogenetischer Syndrome, filigrane klinische Untersuchungstechniken, aber auch grundlegend wichtige apparative Methoden wie EMG und Neurografie. Weiterbildungsordnung und Facharztprüfung müssen auch Instrumente sein, um eine Schmalspurweiterbildung zuverlässig aufzuspüren, und den Finger in die Wunde zu legen, wenn nötig. Der Mehraufwand würde sich meiner Meinung nach lohnen, ansonsten wird man zunehmend „Blogs“ wie den folgenden über die neurologische Facharztprüfung lesen: „Insgesamt wirklich nicht schlimm, ein paar Tage Vorbereitung helfen, Peinlichkeiten zu vermeiden. "Das habe ich zumindest noch keine Studierenden vor dem Staatsexamen sagen hören ...

Christian Gerloff 